\title{
Incidence of Positive Anti-Ro/SSA and Anti-La/SSB Antibodies Among Infants with Prolonged Skin Lesions and Its Relation to Autoimmune Disease in the Mother
}

\author{
Mona Sayed Aly Hassan Makhlouf ${ }^{1}$, Suzan Fahim Ghobashy ${ }^{1}$, Hanan Farouk Mohamed ${ }^{2}$, \\ Ikram Idris Alyan ${ }^{3}$, Fawzy Aly Abualfadl ${ }^{4}$ \\ ${ }^{1}$ Department of Pediatrics, Al Galaa Teaching Hospital, General Organization of Teaching Hospitals and Institutes (GOTHI), Cairo, Egypt \\ ${ }^{2}$ Department of Clinical Pathology, Al Galaa Teaching Hospital, General Organization of Teaching Hospitals and Institutes (GOTHI), Cairo, \\ Egypt \\ ${ }^{3}$ Department of Rheumatology and Rehabilitation, Al Galaa Teaching Hospital, General Organization of Teaching Hospitals and Institutes \\ (GOTHI), Cairo, Egypt \\ ${ }^{4}$ Department of Dermatology, Ahmed Maher Teaching Hospital, General Organization of Teaching Hospitals and Institutes (GOTHI), Cairo, \\ Egypt
}

\section{Email address:}

dr_monamakhlouf@yahoo.com (M. S. A. H. Makhlouf),dr_monamakhlouf2005@hotmail.com (M. S. A. H. Makhlouf), sfg251@yahoo.com (S.F. Ghobashy), hananfarouk@ymail.com (H. F. Mohamed), doc.ikramidris@gmail.com (I. I. Alyan)

\section{To cite this article:}

Mona Sayed Aly Hassan Makhlouf, Suzan Fahim Ghobashy, Hanan Farouk Mohamed, Ikram Idris Alyan, Fawzy Aly Abualfadl. Incidence of Positive Anti-Ro/SSA and Anti-La/SSB Antibodies Among Infants with Prolonged Skin Lesions and Its Relation to Autoimmune Disease in the Mother. International Journal of Immunology. Vol. 6, No. 2, 2018, pp. 30-42. doi: 10.11648/j.iji.20180602.12

Received: June 1, 2018; Accepted: June 26, 2018; Published: July 18, 2018

\begin{abstract}
Many literatures reported that neonatal lupus erythematosus and its associated abnormalities mainly, cutaneous lupus lesions, congenital heart block (CHB), hematologic cytopenias, hepatobiliary disease and cardiomyopathy are associated with anti-Ro/SSA and anti-La/SSB antibodies. With skin findings occur in nearly $95 \%$ of NLE infants, others reported that $100 \%$ of NLE infants with CHB were positive for anti Ro/SSA antibodies and $90.2 \%$ of infants with skin NLE were positive for anti Ro/SSA. And long-term follow-up of children with neonatal lupus and their unaffected siblings revealed that $12.24 \%$ of NLE children identified definite rheumatic/autoimmune diseases after more than 8 years. Aim of the study: The aim of this study was to determine the incidence of positive anti-Ro/SSA and anti-La/SSB antibodies in infants with recurrent prolonged skin lesions and their mothers to determine asymptomatic mothers at risk to develop autoimmune disease. Subjects and methods: a total of 90 infants with recurrent prolonged skin lesions below the age of 6 months and their mothers attending pediatric screening and follow up clinic at Al Galaa Teaching Hospital in Cairo, Egypt were investigated for positivity of anti Ro/SSA and anti La/SSB antibodies using enzyme linked immunoassay (ELISA). Results: Incidence rate of positive anti Ro/SSA alone and anti La/SSB alone antibodies in infants were $34.4 \%$ and $23.3 \%$ respectively, and positive both anti Ro/SSA and anti $\mathrm{La} / \mathrm{SSB}$ antibodies was $21.1 \%$, while in their mothers, positive both anti Ro/SSA and anti La/SSB antibodies was $36.7 \%$. Among the 33 cases with positive antibodies female: male ratio was 2.3:1, and rheumatologic and autoimmune diseases was positive in $14(42.4 \%)$ mothers, while, $19(57.6 \%)$ were asymptomatic mothers, anemia, lymphocytosis, neutropenia with relative lymphocytosis, eosinophilia and thrombocytopenia were found in $6.1 \%, 9.1 \%, 3.0 \%, 9.1 \%$ and $12.1 \%$ respectively. Liver transaminases were elevated in $4(12.1 \%)$. Positive anti Ro/SSA and anti La/SSB antibodies were statistically significantly correlated with infant age, body weight, body length and head circumference, female sex, infants mothers' rheumatologic and autoimmune diseases, distribution of skin lesions, positive history of steroid containing cream use, and thrombocytopenia. However, both antibodies showed no statistically significant correlation with infant feeding, rate of recurrent skin lesions, anemia, lymphocytosis, neutropenia with relative lymphocytosis, eosinophilia, positive CRP, and elevated ESR. Conclusion: our infant cases with prolonged skin lesions with positive anti Ro/SSA and anti La/SSB antibodies with their mothers' especially asymptomatic ones should be investigated and followed up properly for development of NLE and autoimmune diseases.
\end{abstract}


Keywords: Anti Ro/SSA Antibody, Anti La/SSB Antibody, Cutaneous NLE, Autoimmune Diseases

\section{Introduction}

Autoantibodies to DNA and non-DNA antigens occur in patients with multisystem autoimmune disease e.g., systemic lupus erythematosus [SLE], rheumatoid arthritis, and Sjögren syndrome [SS]. Non-DNA antigens include three main categories: the Ro group, the La group and the U group comprising U1-U6 small nuclear RNAs [1-3]. The Ro group contains two major different proteins Ro/SSA: a $52 \mathrm{kDa}$ Ro (with two subtypes alpha and beta) and a $60 \mathrm{kDa}$ Ro. While the La group contains only one La/SSB protein: a $48 \mathrm{kDa} \mathrm{La}$. Anti Ro/SSA antibodies are detected in the sera of $30 \%$ of patients with SLE, even during preclinical setting; and strongly associated (90\%) with some subtypes of SLE, it is also associated with primary Sjögren syndrome (50\% to $60 \%$ ) and with undifferentiated connective tissue disease (UCTD) [4]. NLE encompasses a variety of autoimmune manifestations that results from either congenital trans-placental passage of maternal antinuclear and ribonuclear autoantibodies against the Ro/La RNA-protein complex or to less extent from infant's intrinsic deregulated immune system (Primary infantile SLE). Anti-Ro and anti-La autoantibodies targeting fetal and neonatal tissues (Neonatal Lupus Syndromes) are actively transported into the fetal circulation, leading to clinical manifestations that include potentially life-threatening congenital heart block [5 - 7]. It occurs in infants born to mothers with rheumatic conditions including, but not restricted to, systemic lupus erythematosus. Most clinical manifestations of NLE resolve spontaneously with vanishing of maternal auto-antibodies from the neonatal circulation except congenital complete heart block [8]. However, only $1 \%$ of infants with positive maternal autoantibodies develop neonatal lupus erythematosus and some cases are born to asymptomatic mothers who are unaware of their autoimmune disorders and get their seropositivity discovered after an affected infant. Maternal disease activity during pregnancy has been associated with increased frequency of neonatal morbidities and admission to the NICU [7]. Cimaz et al., in their studied 128 infants born from 124 pregnancies in 112 women with anti-Ro antibodies with or without anti-La antibodies. They reported 2 cases $(1.6 \%)$ of congenital heart block (CHB) and 21 children $(16 \%)$ developed cutaneous NLE, with laboratory testing showed hematologic abnormalities in $27 \%$ of the babies and elevation of liver enzymes in $26 \%$ [9].

Sontheimer et al, found that skin findings occur in nearly $95 \%$ of their NLE infants. The eruption, which can occur at birth but more typically within a few weeks after birth, is similar to the lesions of subacute cutaneous lupus erythematosus [10]. The skin eruptions typically, erythematous scaling and annularpolycyclic plaques appear on sun-exposed areas, especially on the head and neck, and often in a malar distribution [11], also, similar plaques may appear on the trunk or extremities [12].

Wei Sun et al, found $100 \%$ of NLE infants with CHB were positive for anti Ro/SSA antibodies and 90.2\% (46/51) of infants with skin NLE were positive for anti Ro/SSA [12]. Furthermore, positive anti-Ro and anti-La and hepatic and hematological abnormalities are common in cases with skin involvement [11].

Martin's et al, in their study for long-term follow-up of children with neonatal lupus and their unaffected siblings, they reported that $12.24 \%(6 / 49)$ of NLE children identified definite rheumatic/autoimmune diseases after more than 8 years suggesting that prior to adolescence children with NLE require continued follow-up [13].

The current study hypothesized that autoimmune pathogenic process and connective tissue disorders e.g., NLE, could be a cause for recurrent prolonged skin lesions in infants especially below the age of 6 months. The aim of this study was to determine the incidence of positive anti-Ro/SSA $(60 \mathrm{kDa}$ and $52 \mathrm{kDa}$ ) and anti-La/SSB antibodies (being are directly involved in the pathogenesis of NLE and other connective tissue disorders), among infants with recurrent prolonged skin lesions attending pediatric screening and follow up clinic at Al Galaa Teaching Hospital in Cairo, Egypt. At the same time to determine the incidence in their mothers to determine asymptomatic mothers at risk to develop autoimmune disease.

\section{Subjects and Methods}

\subsection{Subjects}

A total of 90 infants with recurrent prolonged skin eruptions and their mothers from those attending pediatric screening and follow up clinic at Al Galaa Teaching Hospital, in Cairo, were enrolled in this study during 12 moths started from March 2017 till March 2018. Inclusion criteria were: Age less than 6 months and Infants with recurrent prolonged skin lesions more than 2 weeks duration in the form of annular-polycyclic erythematous scaling plaques, in face, head, trunk and extremities, Exclusion criteria were: Age more than 6 months, benign neonatal skin lesions and skin lesions less than 2 weeks duration.

\subsection{Methods}

All participants were subjected to: Full history taking and thorough clinical examination. Electrocardiogram to infants to detect cardiac abnormalities, and Laboratory tests including: complete blood count and differential count using Cell Tac, liver profile by Hitachi 912, erythrocyte sedimentation rate and C-reactive protein, and Measurements of anti Ro/SSA and anti La/SSB for both infants and their mothers by ELISA. A blood sample of $7 \mathrm{ml}$ was obtained by venipuncture from infants, $2 \mathrm{ml}$ were put on EDTA to perform $\mathrm{CBC}$, ESR and $5 \mathrm{ml}$ were left to clot and sera obtained were used for liver profiles, CRP, and the rest were stored frozen at -20 until assay of anti Ro/SSA and anti $\mathrm{La} / \mathrm{SSB}$. Another blood sample of $3 \mathrm{ml}$ was obtained from mothers, left to clot and sera obtained were stored frozen at 20 until assay of anti Ro/SS-A and anti La/SS-B. 


\subsection{Assay of Anti Ro/SS-A and Anti La/SS-B}

QUANTA Lite SS-B ELISA for the semi-quantitative detection of SS-B antibodies in human serum (INOVA Diagnostics, Inc., cat no 708575).

QUANTA Lite SS-A ELISA for the semi-quantitative detection of SS-A $(60 \mathrm{kDa}$ and $52 \mathrm{kDa})$ antibodies in human serum (INOVA Diagnostics, Inc., cat no 708570).

Principles of the two Procedures:

Purified SS-B (or SS-A) antigen is bound to the wells of a polystyrene microwell plate under conditions that will preserve the antigen in its native state. Pre-diluted controls and diluted patient sera are added to separate wells; allowing any SS-B or SS-A antibodies present to bind to the immobilized antigen. Unbound sample is washed away and an enzyme labeled anti-human IgG conjugate is added to each well. A second incubation allows the enzyme labeled anti-human IgG to bind to any patient antibodies, which have become attached to the microwells. After washing away any unbound enzyme labeled anti-human $\mathrm{IgG}$, the remaining enzyme activity is measured by adding a chromogenic substrate and measuring the intensity of the color that develops. The absorbance (OD) of each well is read at 450 $\mathrm{nm}$ within one hour of stopping the reaction with $620 \mathrm{~nm}$ that are used as reference wave length.

Calculation of Results:

The reactivity for each sample can be calculated by dividing the OD of the sample by the OD of the SS-A (or SS-B) ELISA low positive. The result is then multiplied by the number of units assigned to the SS-B (or SS-A) low positive found on the label.

$$
\begin{gathered}
\text { Sample value (units) }=\frac{\text { Sample oD }}{\text { SS-B ELISA low positive (OD)(Or SS-A) }} \times \\
\text { SS - B ELISA low positive (units) (or SS - A) }
\end{gathered}
$$

Interpretation of Results:

The ELISA assay was very sensitive to technique and capable of detecting even small differences in patient

populations.

The sample was classified as negative, weak positive, moderate positive or strong positive according to the table below:
Negative
Weak positive
Moderate positive
Strong positive

\subsection{Statistical Analysis}

Results were reported as mean \pm standard deviation (SD) for the quantitative variables and percentages for the categorical variables. The univariate Pearson's chi-square test was used to measure the correlation of each variable with result of positive anti Ro/SS-A and anti La/SS-B in both infants and mothers. Pvalues of 0.05 or less were considered statistically significant. All statistical analyses were performed using the statistical package for social science (SPSS) version 20.

\section{Results}

Ninety infants with recurrent prolonged skin lesions and their mothers were enrolled in this study, [53 females and 37 males, with female: male ratio $1.43: 1$, their mean age $=3.23$ \pm 1.461 months (mean \pm SD) $]$ and their mothers mean age $=$ $27.13 \pm 4.913$ years (mean $\pm \mathrm{SD}$ ). Infants`anthropometric measures were within normal percentiles for age, [BW was $4.52 \pm 1.00 \mathrm{~kg}$, length was $59.36 \mathrm{~cm} \pm 4.37$ and head circumference was $39.66 \pm 2.23 \mathrm{~cm}$ ]. All studied infants had no cardiac symptoms and infants` electrocardiograms (ECG) were normal. The quantitative variables in the studied infants including age of infants and mothers, infant anthropometric measures, and result of laboratory tests including basic laboratory tests and Anti Ro/SSA $(60 \mathrm{kDa}$ and $52 \mathrm{kDa})$ and anti $\mathrm{La} / \mathrm{SSB}$ antibodies are shown in Table 1.

\begin{tabular}{|c|c|c|c|c|}
\hline \multicolumn{2}{|l|}{ Variables } & Range & Mean & Std. Deviation \\
\hline \multicolumn{2}{|c|}{ Infant Age (months) } & $1-6$ & 3.23 & 1.46 \\
\hline \multicolumn{2}{|c|}{ Mother age (years) } & $20-37$ & 27.13 & 4.91 \\
\hline \multicolumn{5}{|c|}{ Infants anthropometric measures: } \\
\hline \multicolumn{2}{|l|}{ BW (kg) } & $3.000-8.000$ & 4.52 & 1.00 \\
\hline \multicolumn{2}{|l|}{ Length $(\mathrm{Cm})$} & $54.00-70.00$ & 59.36 & 4.37 \\
\hline \multicolumn{2}{|c|}{ Head Circumference (Cm) } & $36.50-45.00$ & 39.66 & 2.23 \\
\hline \multicolumn{5}{|l|}{ Laboratory tests: } \\
\hline \multicolumn{2}{|l|}{$\operatorname{ESR}(\mathrm{mm})$} & $8-24$ & 14.43 & 4.07 \\
\hline \multicolumn{2}{|c|}{ Infant Hemoglobin $(\mathrm{gm} / \mathrm{dl})$} & $8.80-12.20$ & 11.00 & 1.012 \\
\hline \multicolumn{2}{|c|}{ Total WBCs (thousands/cmm) } & $4370-11500$ & 7870.89 & 2125.64 \\
\hline \multicolumn{2}{|c|}{ Neutrophils } & $1380-6260$ & 4117.43 & 1173.81 \\
\hline \multicolumn{2}{|l|}{ Eosinophils } & $50-752$ & 150.74 & 115.35 \\
\hline \multicolumn{2}{|l|}{ Lymphocytes } & $2045-6498$ & 3613.16 & 979.77 \\
\hline \multicolumn{2}{|c|}{ Infant Platelets (thousands/cmm) } & $75000-411000$ & 260566.67 & 78810.40 \\
\hline \multirow[t]{2}{*}{ Infants: } & Anti Ro/SSA(U) & $2.60-53.10$ & 18.54 & 19.04 \\
\hline & Anti La/SSB (U) & $2.00-47.90$ & 12.45 & 15.29 \\
\hline \multirow[t]{2}{*}{ Mothers: } & Anti Ro/SSA(U) & $2.10-49.60$ & 18.49 & 17.62 \\
\hline & Anti La/SSB (U) & $1.70-53.30$ & 15.76 & 17.63 \\
\hline
\end{tabular}

Table 1. Basic quantitative variables among all studied cases. 
Incidence rate of positive anti Ro/SSA and anti La/SSB in infants and mothers was calculated as overall incidence rate of positive antibodies per 100 studied infants and mothers during the study period using the following formula:

$$
\begin{aligned}
& \frac{\text { No of positive anti Ro/SSA antibodies in infants }}{\text { Total no of studies infants during } 12 \text { months }} \times 100=\frac{31 \times 100}{90}=34.4 \% \text { per } 100 \text { studied infants } \\
& \frac{\text { No of positive anti La/SSB antibodies in infants }}{\text { Total no of studies infants during } 12 \text { months }} \times 100=\frac{21 \times 100}{90}=23.3 \% \text { per } 100 \text { studied infants } \\
& \frac{\text { No of positive both antibodies in infants }}{\text { Total no of studies infants during } 12 \text { months }} \times 100=\frac{19 \times 100}{90}=21.1 \% \text { per } 100 \text { studied infants }
\end{aligned}
$$

As 33 mothers had positive both antibodies, the incidence rate in the mothers was

$$
\frac{\text { No of positive both antibodies in mothers }}{\text { Total no of studied mothers during } 12 \text { months }} \times 100=\frac{33 \times 100}{90}=36.7 \% \text { per } 100 \text { studied mothers }
$$

Table 2 and 3 demonstrates the frequency of different variables in all studied infants and within infants with positive anti Ro/SSA and anti La/SSB antibodies. Total of 33 (36.7.2\%) infants and their mothers were positive for anti $\mathrm{Ro} / \mathrm{SSA}$ and anti La/SSB antibodies by ELISA, from whom 19 infants $(57.5 \%)$ were positive for both antibodies, 12 cases $(36.4 \%)$ were positive for anti Ro/SSA alone, and 2 infants $(6.1 \%)$ were positive for anti La/SSB alone with total of $31(93.9 \%)$ infant positive for anti Ro/SSA antibody and $21(63.6 \%)$ infant positive for anti La/SSB antibody as shown in table 3. Within the studies infant 52 cases $(57.8 \%)$ were breast fed, while, $20(22.2 \%)$ and $18(20 \%)$ cases were formula fed alone and breast fed with formula supplement respectively, The studied infants had positive mother medical diseases in $26(28.9 \%)$ cases, e.g., diabetes mellitus in 5 $(5.6 \%)$ cases, hypertension in $3(3.3 \%)$ cases, and atopic dermatitis in 4 (4.4\%) cases. Rheumatologic and autoimmune diseases in $14(15.5 \%)$ cases including $5(5.6 \%)$ cases of systemic lupus erythematosus, 4 (4.4\%) cases of rheumatoid arthritis, $2(2.2 \%)$ cases of Psoriasis and $3(3.3 \%)$ cases of rheumatic fever. Regarding the skin lesions, the distribution of skin lesions among the studied infants were involving one site alone e.g., head, extremities, trunk, or involving more than one site e.g., head and trunk, head and extremities, trunk and extremities or head, trunk and extremities all together with frequency of distribution are shown in table 2 and figure 1 showing some photos for infants with positive antibodies, the skin lesions in all studied infant showed erythema, scaling and crusting.

The recurrence rate of skin lesions were twice in 36 $(40 \%)$ cases, thrice in $39(43.3 \%)$ and more than three times in $15(16.7 \%)$ cases. Survival rate was $100 \%$ and resolving skin lesions rate was $100 \%$ with Conservative care and Avoid ultraviolet light exposure with History of use of Skin creams containing steroid was positive in 47 $(52.2 \%)$ cases. Frequency of positive finding of anemia, lymphocytosis, neutropenia with relative lymphocytosis, eosinophilia and thrombocytopenia were $4.4 \%, 3.3 \%$, $2.2 \%, 3.3 \%$, and $4.4 \%$ respectively in studied infants.
Acute phase reactant including C-reactive protein (CRP) was positive in $35(38.9 \%)$ cases and erythrocyte sedimentation rate (ESR) was elevated in $47(52.2 \%)$ cases. Liver transaminases, alanine transaminase (ALT) and aspartate transaminase (AST) were elevated in 4 (4.4\%) cases among studied infants.

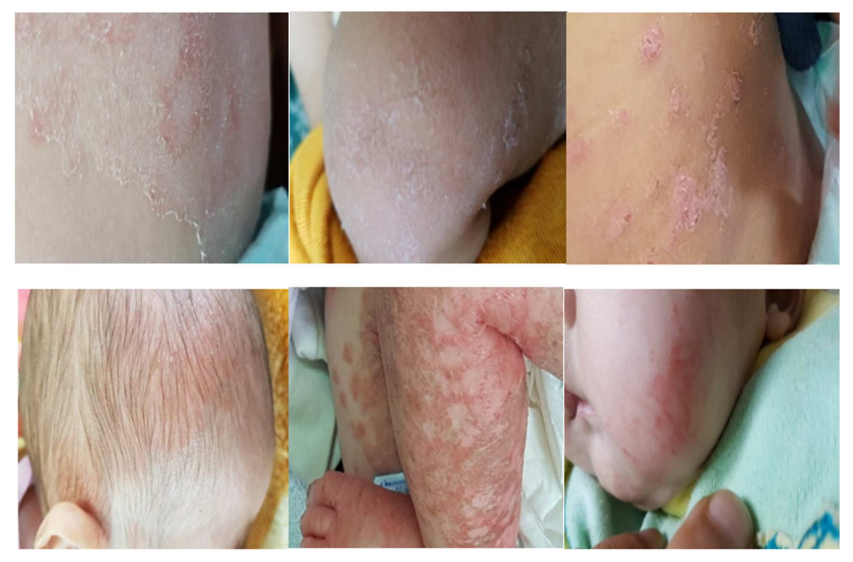

Figure 1. Sample photos for some infants with positive anti Ro/SSA and anti La/SSB antibodies.

Among the 33 cases with positive antibodies, 10 cases (30.3\%) were males and 23 cases $(69.7 \%)$ were females, with female: male ratio $2.3: 1$, this indicates that higher rate of positive antibodies occurs in female infants. Infants with positive antibodies had no difference regarding breast or formula feeding with formula fed in $12(36.4 \%)$ cases, breast fed with formula supplement, in $10(30.3 \%)$ cases and breast fed in $11(33.3 \%)$ cases. Infants' mother medical diseases in cases with positive antibodies showed positive history for atopic dermatitis in $4(12.1 \%)$ cases, while, positive rheumatologic and autoimmune diseases in $14(42.4 \%)$ cases including $5(15.2 \%)$ cases of systemic lupus erythematosus, $4(12.1 \%)$ cases of rheumatoid arthritis, $2(6.1 \%)$ cases of Psoriasis and $3(9.1 \%)$ cases of rheumatic fever, figure 2 . 


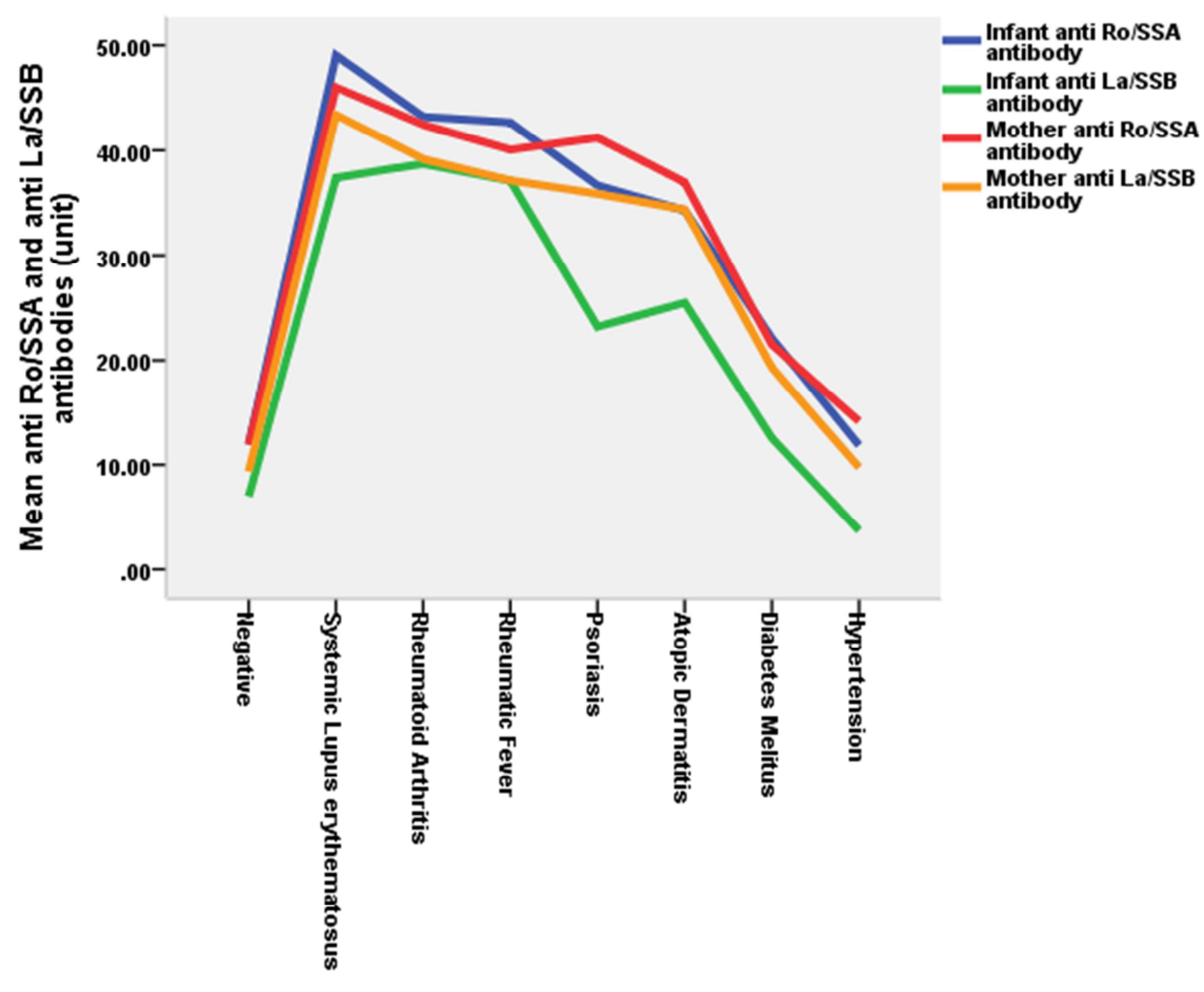

Infants' mothers medical diseases

Figure 2. Relation of anti Ro/SSA and anti La/SSB antibodies to infants ' mothers medical diseases.

Regarding skin lesions, infants with positive antibodies had higher frequency for distribution in more than one site being $10(30.3 \%)$ in head and extremities, $13(39.4 \%)$ in head and trunk, $4(12.1 \%)$ in trunk and extremities, and $6(18.2 \%)$ in head, trunk and extremities figure 3, with frequency for recurrent skin lesions rate being thrice in $16(48.5 \%)$, twice in $9(27.3 \%)$ and more than three times in $8(24.2 \%)$ cases. figure 4.

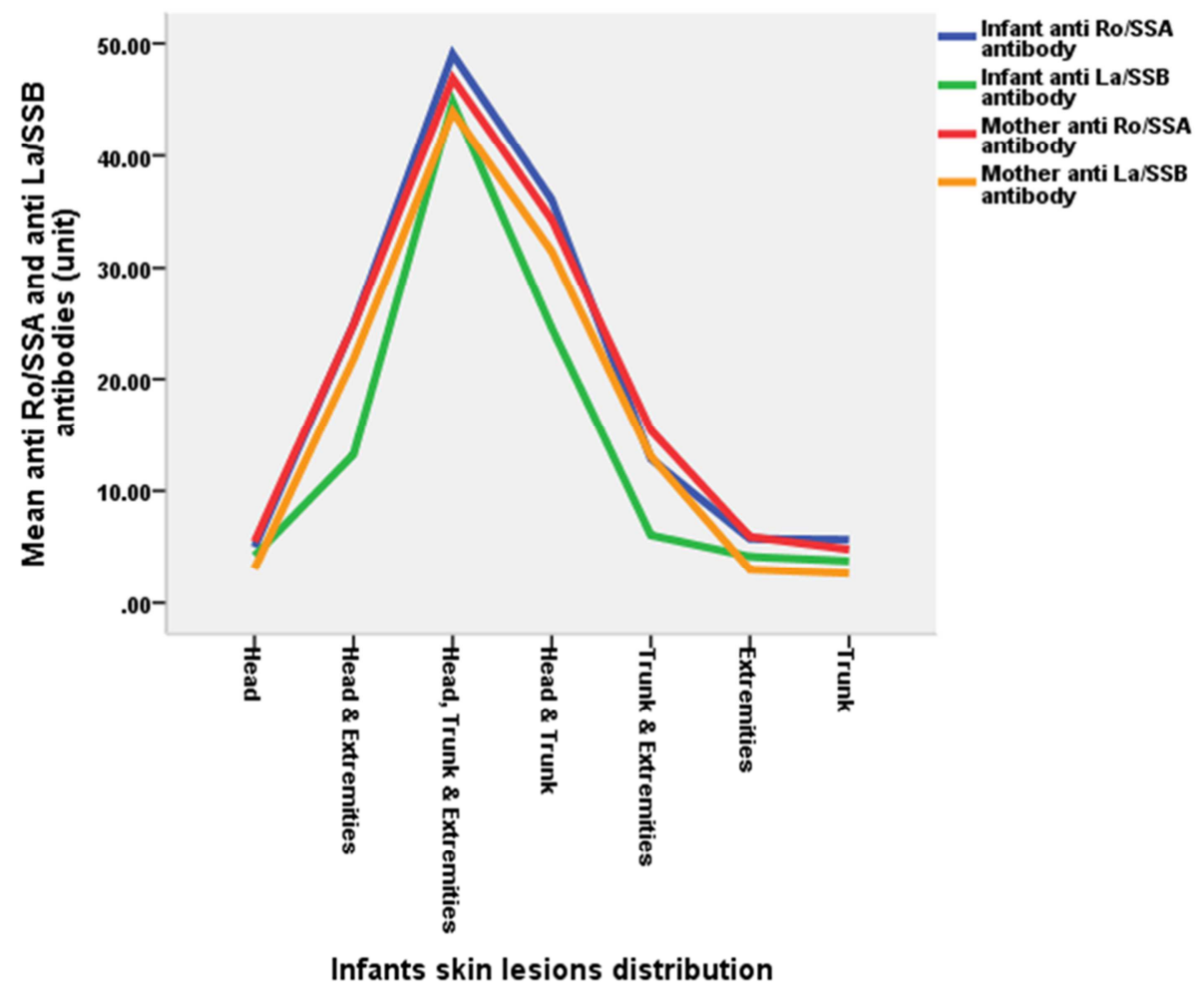

Figure 3. Relation of anti Ro/SSA and anti La/SSB antibodies to infants `skin lesions distribution. 


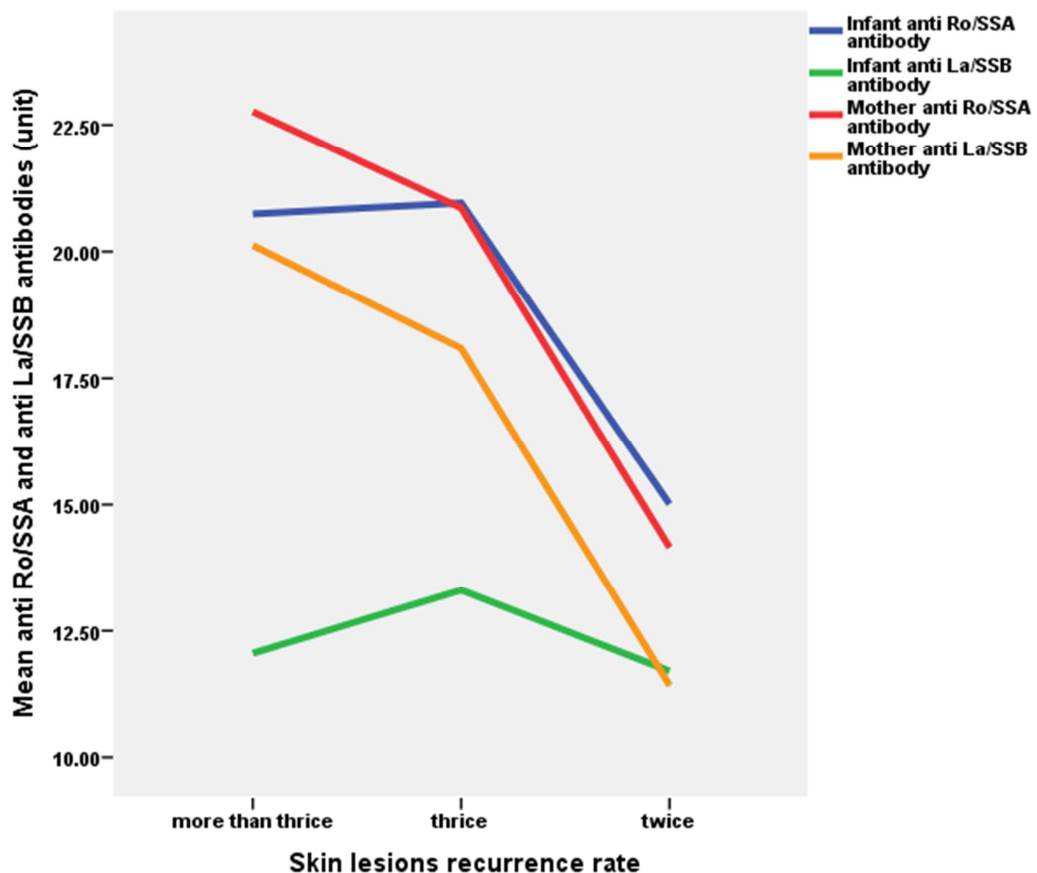

Figure 4. Relation of anti Ro/SSA and anti La/SSB antibodies to rate of infants 'skin lesions recurrence.

Frequency of positive finding of anemia, lymphocytosis, neutropenia with relative lymphocytosis, eosinophilia and thrombocytopenia were $6.1 \%, 9.1 \%, 3.0 \%, 9.1 \%$ and $12.1 \%$ respectively in infants with positive antibodies. Acute phase reactant including C-reactive protein (CRP) was positive in
$18(54.5 \%)$ cases and erythrocyte sedimentation rate (ESR) was elevated in $17(51.5 \%)$ cases. Liver transaminases, alanine transaminase (ALT) and aspartate transaminase (AST) were elevated in $4(12.1 \%)$ cases among studied infants, tables 2 and 3.

Table 2. Frequency of qualitative variables among all studied cases and positive Anti Ro/SSA and Anti La/SSB cases.

\begin{tabular}{|c|c|c|c|c|c|}
\hline \multirow{2}{*}{ Variables } & & \multicolumn{2}{|c|}{ Among all studied cases } & \multicolumn{2}{|c|}{ Among positive cases } \\
\hline & & Frequency & Percent & Frequency & Percent \\
\hline \multirow{2}{*}{ Sex } & Female & 53 & 58.9 & 23 & 69.7 \\
\hline & Male & 37 & 41.1 & 10 & 30.3 \\
\hline \multirow{3}{*}{ Infant Feeding } & Breast and formula fed & 18 & 20 & 10 & 30.3 \\
\hline & Breast & 52 & 57.8 & 11 & 33.3 \\
\hline & Formula & 20 & 22.2 & 12 & 36.4 \\
\hline \multirow{10}{*}{ Mother Diseases } & Negative & 64 & 71.1 & 12 & 36.4 \\
\hline & Positive & 26 & 28.9 & 21 & 63.6 \\
\hline & Diabetes Mellitus & 5 & 5.6 & 2 & 6.1 \\
\hline & Hypertension & 3 & 3.3 & 1 & 3 \\
\hline & Atopic dermatitis & 4 & 4.4 & 4 & 12.1 \\
\hline & Rheumatologic diseases & 14 & 15.5 & 14 & 42.4 \\
\hline & Systemic Lupus erythematosus & 5 & 5.6 & 5 & 15.2 \\
\hline & Rheumatoid Arthritis & 4 & 4.4 & 4 & 12.1 \\
\hline & Psoriasis & 2 & 2.2 & 2 & 6.1 \\
\hline & Rheumatic Fever & 3 & 3.3 & 3 & 9.1 \\
\hline \multirow{7}{*}{ Site of skin lesions } & Extremities & 17 & 18.9 & - & - \\
\hline & Head & 14 & 15.6 & - & - \\
\hline & Head and Extremities & 17 & 18.9 & 10 & 30.3 \\
\hline & Head and Trunk & 16 & 17.8 & 13 & 39.4 \\
\hline & Head, Trunk and Extremities & 6 & 6.7 & 6 & 18.2 \\
\hline & Trunk & 7 & 7.8 & - & - \\
\hline & Trunk and Extremities & 13 & 14.4 & 4 & 12.1 \\
\hline \multirow{3}{*}{ Number of recurrence } & More than 3 times & 15 & 16.7 & 8 & 24.2 \\
\hline & thrice & 39 & 43.3 & 16 & 48.5 \\
\hline & twice & 36 & 40.0 & 9 & 27.3 \\
\hline \multirow{2}{*}{ Use of steroids creams } & Free & 43 & 47.8 & 9 & 27.3 \\
\hline & Steroid & 47 & 52.2 & 24 & 72.7 \\
\hline \multirow{2}{*}{ Outcome } & Survival & 90 & 100 & 33 & 100 \\
\hline & Resolving skin lesions & 90 & 100 & 33 & 100 \\
\hline Total number of cases & & 90 & 100 & 33 & 100 \\
\hline
\end{tabular}


Mona Sayed Aly Hassan Makhlouf et al.: Incidence of Positive Anti-Ro/SSA and Anti-La/SSB Antibodies Among Infants with Prolonged Skin Lesions and Its Relation to Autoimmune Disease in the Mother

Table 3. Frequency of laboratory variables among all studied cases and positive Anti Ro/SSA and Anti La/SSB antibodies cases.

\begin{tabular}{|c|c|c|c|c|c|}
\hline \multirow{2}{*}{ Variables } & & \multicolumn{2}{|c|}{ Among all studied cases } & \multicolumn{2}{|c|}{ Among positive cases } \\
\hline & & Frequency & Percent & Frequency & Percent \\
\hline \multirow{5}{*}{$\mathrm{CBC}$} & Anemia & 4 & 4.4 & 2 & 6.1 \\
\hline & Eosinophilia & 3 & 3.3 & 3 & 9.1 \\
\hline & Lymphocytosis & 3 & 3.3 & 3 & 9.1 \\
\hline & Neutropenia with relative lymphocytosis & 2 & 2.2 & 1 & 3.0 \\
\hline & Thrombocytopenia & 4 & 4.4 & 4 & 12.1 \\
\hline \multirow{2}{*}{ CRP } & Negative & 55 & 61.1 & 15 & 45.5 \\
\hline & Positive & 35 & 38.9 & 18 & 54.5 \\
\hline \multirow{2}{*}{ ESR } & Normal & 43 & 47.8 & 16 & 48.5 \\
\hline & Elevated & 47 & 52.2 & 17 & 51.5 \\
\hline Liver enzymes & Normal & 86 & 95.6 & 29 & 87.9 \\
\hline \multirow{2}{*}{ ALT } & Elevated & 4 & 4.4 & 4 & 12.1 \\
\hline & Normal & 86 & 95.6 & 29 & 87.9 \\
\hline \multirow[t]{2}{*}{ AST } & Elevated & 4 & 4.4 & 4 & 12.1 \\
\hline & Negative & 59 & 65.6 & 2 & 6.1 \\
\hline \multirow{5}{*}{ Infant anti Ro/SSA } & Positive & 31 & 34.4 & 31 & 93.9 \\
\hline & Alone & 12 & 13.3 & 12 & 36.4 \\
\hline & With anti $\mathrm{La} / \mathrm{SSB}$ & 19 & 21.1 & 19 & 57.5 \\
\hline & Moderate positivity & 23 & 25.6 & 23 & 69.7 \\
\hline & Weak positivity & 8 & 8.9 & 8 & 24.2 \\
\hline \multirow{6}{*}{ Infant anti $\mathrm{La} / \mathrm{SSB}$} & Negative & 69 & 76.7 & 12 & 36.4 \\
\hline & Positive & 21 & 23.3 & 21 & 63.6 \\
\hline & Alone & 2 & 2.2 & 2 & 6.1 \\
\hline & With anti Ro/SSA & 19 & 21.1 & 19 & 57.5 \\
\hline & Moderate positivity & 15 & 16.7 & 15 & 45.5 \\
\hline & Weak positivity & 6 & 6.7 & 6 & 18.2 \\
\hline \multirow{4}{*}{ Mother Anti Ro/SSA } & Negative & 57 & 63.3 & 0 & 0 \\
\hline & Positive & 33 & 36.7 & 33 & 100.0 \\
\hline & Moderate positivity & 22 & 24.4 & 22 & 66.7 \\
\hline & Weak positivity & 11 & 12.2 & 11 & 33.3 \\
\hline \multirow{4}{*}{ Mother Anti La/SSB } & Negative & 57 & 63.3 & 0 & 0 \\
\hline & Positive & 33 & 36.7 & 33 & 100.0 \\
\hline & Moderate positivity & 22 & 24.4 & 22 & 66.7 \\
\hline & Weak positivity & 11 & 12.2 & 11 & 33.3 \\
\hline Total number of cases & & 90 & 100 & 33 & 100 \\
\hline
\end{tabular}

Table 4. Correlation of positive anti Ro/SSA and anti La/SSB antibodies with different infants studied variables.

\begin{tabular}{lllll}
\hline \multirow{2}{*}{ Variables } & \multicolumn{2}{l}{ Positive Anti Ro/SSA antibody } & \multicolumn{2}{l}{ Positive anti La/SSB antibody } \\
\cline { 2 - 5 } & Pearson Correlation & Sig. (2-tailed) & Pearson Correlation & Sig. (2-tailed) \\
\hline Infant age & $-.249^{*}$ & .018 & $-.257^{*}$ & .015 \\
Mother age & .105 & .327 & $.225^{*}$ & .033 \\
Female sex & $-.240^{*}$ & .023 & $-.294^{*}$ & .005 \\
BW & $-.289^{* *}$ & .006 & $-.258^{*}$ & .014 \\
Length & $-.275^{*}$ & .009 & $-.274^{* *}$ & .009 \\
HC & $-.262^{*}$ & .013 & $-.260^{*}$ & .013 \\
Type of Infants feeding & -.126 & .028 & .878 \\
Mother rheumatologic and autoimmune diseases & $.390^{*}$ & .484 & .000 \\
Distribution of skin lesions & $.481^{* *}$ & .000 & $.361^{* *}$ & .000 \\
Rate of recurrent skin lesions & .131 & .000 & $.421^{* *}$ & .841 \\
Steroid cream use & $.789^{* *}$ & .218 & .021 & .019 \\
Infant hemoglobin & .179 & .000 & $.246^{*}$ & .050 \\
Total WBCs count & -.047 & .092 & .207 & .576 \\
Neutropenia & -.097 & .657 & -.060 & .433 \\
Eosinophilia & .122 & .361 & -.084 & .116 \\
Lymphocytosis & -.009 & .252 & .167 & .604 \\
Thrombocytopenia & $.297^{* *}$ & .933 &. .055 & .017 \\
CRP & -.187 & .005 & $.250^{*}$ & .219 \\
ESR & -.146 & .298 & .220 & .399 \\
ALT & $.340^{* *}$ & .417 &. .152 & .000 \\
AST & $.340^{* *}$ & .001 & $.462^{* *}$ & .000 \\
Infant anti Ro/SSA & - & .001 & $.462^{* *}$ & $.789^{* *}$ \\
\hline
\end{tabular}




\begin{tabular}{lllll}
\hline \multirow{2}{*}{ Variables } & \multicolumn{2}{l}{ Positive Anti Ro/SSA antibody } & \multicolumn{2}{c}{ Positive anti La/SSB antibody } \\
\cline { 2 - 5 } & Pearson Correlation & Sig. (2-tailed) & Pearson Correlation & Sig. (2-tailed) \\
\hline Infant anti La/SSB & $.789^{* *}$ & .000 & - & - \\
Mother anti Ro/SSA & $.965^{* *}$ & .000 & $.768^{* *}$ & .000 \\
Mother anti La/SSB & $.964^{* *}$ & .000 & $.762^{* *}$ & .000 \\
\hline
\end{tabular}

**. Correlation is significant at the 0.01 level (2-tailed).

*. Correlation is significant at the 0.05 level (2-tailed).

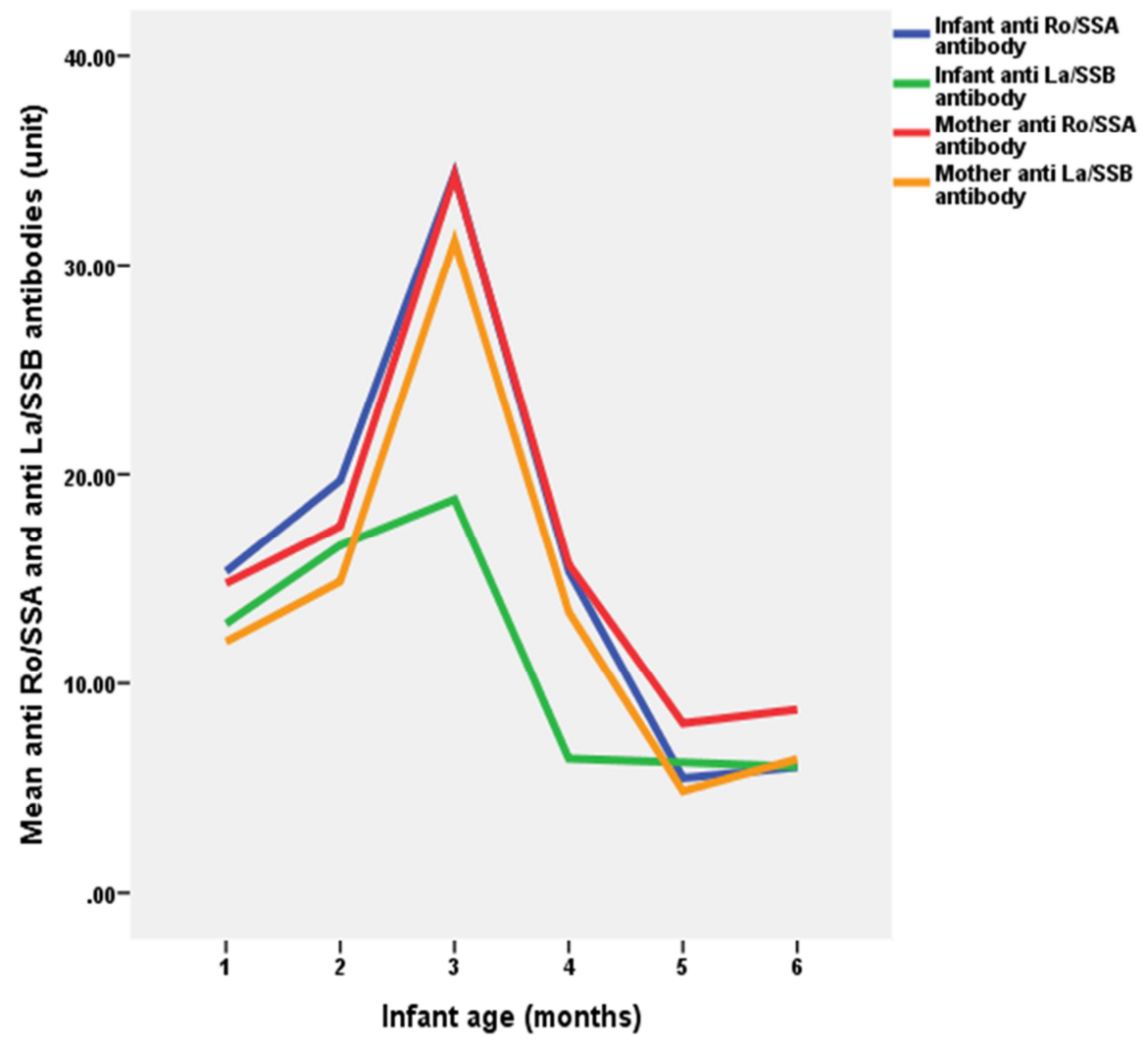

Figure 5. Relation of anti Ro/SSA and anti La/SSB antibodies to infants 'age.

In Table 4 Positive both anti Ro/SSA and anti La/SSB antibodies in studied infants revealed statistically significant negative correlation with infants' age, ( $\mathrm{p}$ value .018, and .015 respectively), (figure 5), body weight, body length and head circumference, ( $\mathrm{p}$ values were .006, .014, .009, and .013, respectively) being elevated at younger age range from 2 to 4 months with highest elevation at age of 3 months, the body weight, length and head circumference showed same correlation as they intend to increase normally with advance of age. Also, revealed statistically significant correlation with female sex ( $p$ value was .023 and .005), however, anti La/SSB antibody showed statistically significant positive correlation with mother age ( $\mathrm{p}$ value .033), but anti Ro/SSA antibody showed no statistically significant correlation ( $\mathrm{p}$ value .327 ). Also, both antibodies showed no statistically significant correlation with type of infant feeding either breast or formula feeding, ( $p$ value .484 and .878) and showed statistically significant positive correlation with infants' mother medical diseases specially rheumatologic and autoimmune diseases, ( $p$ value .000). Both antibodies also, revealed statistically significant correlation with distribution of skin lesions that involving more than one site and positive history of steroid containing cream use, ( $p$ value .000 and .019) respectively, however, no statistically significant correlation was found regarding rate of recurrent skin lesions, ( $p$ value .218 and .841$)$. Positive antibodies revealed statistically significant correlation with thrombocytopenia, ( $p$ value .005 and .017) but, no statistically significant correlation with anemia $(\mathrm{p}$ value .092 and .050), lymphocytosis, (p value .933 and .604) neutropenia with relative lymphocytosis, ( $p$ value .361 and .433) and eosinophilia, ( $\mathrm{p}$ value .252 and .116). Regarding acute phase reactants both antibodies revealed no statistically significant correlation with positive CRP, and elevated ESR ( $p$ values were .298, .219 and .417, .399 respectively), and statistically significant correlation was found with elevated liver transaminases, ( $\mathrm{p}$ value .001 and .000). Infant Anti Ro/SSA antibody alone, showed statistically significant correlation with infant anti La/SSB antibody and both mothers antibodies, ( $p$ value .000), the same statistically significant correlation was found regarding infant anti $\mathrm{La} / \mathrm{SSB}$ antibody alone, with infant anti Ro/SSA antibody and mothers' antibodies. 


\section{Discussion}

Autoimmune diseases are a group of heterogeneous disorders in which the immune system attacks against its own cells, tissues or organs, resulting in an auto inflammatory reaction that could be cytokines/protein mediated, or antibodies mediated with resultant multiple clinical and laboratory aspects [14]. Diseases are more frequent in female gender than in male (78\%). Autoantibody mediated diseases can influence the fetus and newborn, because they are from Immunoglobulin $\mathrm{G}(\mathrm{IgG})$ type which can cross the placenta and enter the fetal circulation [15]. Many literatures reported that neonatal lupus erythematosus and its associated abnormalities mainly, cutaneous lupus lesions, congenital heart block (CHB), hematologic cytopenias, hepatobiliary disease and cardiomyopathy are associated with anti-Ro/SSA and anti-La/SSB antibodies [16 - 19]. Neonatal Lupus Syndrome (NLS) in infants is a major outcome of mothers with systemic lupus erythematosus that has been reported in many studies [20 - 26]. It is an acquired autoimmune passive disease mediated by maternal antibodies, reported in about 1 $2 \%$ of infants born to mothers with autoimmune disease with Anti-Ro/SSA, and Anti-La/SSB antibodies [25]. However, some cases occur in children of mothers who have the same autoantibodies but have no symptoms of SLE, and no other autoimmune disease during pregnancy [27]. The pathogenesis of the disease is not completely clear, but is likely to be greater than the simple passage of paired antibodies. It is also necessary that all lupus patients who decide to have a pregnancy should be screened for AntiRo/SSA, and Anti-La/SSB. Identifying mothers at risk allows quick treatment before or after birth [28].

This study reported a female: male ratio 2.3:1 with female predominance which was statistically correlated with anti $\mathrm{Ro} / \mathrm{SSA}$ and $\mathrm{La} / \mathrm{SSB}$ antibodies in studied infants with recurrent prolonged skin lesions who had positive antibodies, this result is with concordance to results of studies done by Weston et al, Dickey et al, and Dörner et al, who reported that female neonates with NLE had increased incidence of rash, with a female-to-male ratio ranging between 2: 1 and 3: 1 [11, 29, 30], however, the current study result is in disconcordance with result of $\mathrm{Li}$ et al [31], who reported a ratio of female: male 1.05: 1 with no female predominance and Buyon \& Clancy who reported that $55 \%$ of studied infants with skin rash were females with no female predominance in a study based on data obtained from the large United States national Research Registry of Neonatal Lupus (RRNL) [32]. On the other side Peñate et al and Asboth et al reported that cutaneous NLE more common in male than female babies $[33,34]$.

In the current study to assess the incidence of anti Ro/SSA and anti La/SSB antibodies in infants who had recurrent prolonged skin lesions 33/90 cases (36.7) with their mothers had positive anti Ro/SSA and anti La/SSB antibodies, with 19 infants $(57.5 \%)$ of them were positive for both antibodies, 12 cases $(36.4 \%)$ were positive for anti Ro/SSA alone, and 2 infants $(6.1 \%)$ were positive for anti $\mathrm{La} / \mathrm{SSB}$ alone, a result that is near to result of study done by Wei Sun et al, who found that infants involved in their study with skin NLE were positive for anti Ro/SSA antibody alone in 18 cases $(33.33 \%)$, anti $\mathrm{La} / \mathrm{SSB}$ antibody alone in 1 case $(1.85 \%)$, and both antibodies in 30 cases (55.56\%) [12].

The studied infants with positive antibodies had negative mother history for rheumatologic and autoimmune diseases in $19(57.6 \%)$ and positive history in $14(42.4 \%)$ cases, who had systemic lupus erythematosus in $5(15.2 \%)$ cases, rheumatoid arthritis in $4(12.1 \%)$, Psoriasis in $2(6.1 \%)$ and 3 $(9.1 \%)$ cases of rheumatic fever, this result is in agreement with Wei Sun et al, who found that 24 mothers (44.44\%) in their study for infants with skin NLE were asymptomatic, inspite that they were positive for same antibodies as their infants with 30 cases $(55.56 \%)$ had rheumatologic and autoimmune diseases, (27 cases) had SLE, (1 case) had rheumatoid arthritis, 1 case had Sjögren syndrome. and 1 case. had psoriasis, but the current study have less cases of SLE and no diagnosed cases of Sjögren syndrome [12], also, the study result regarding percentage of asymptomatic mothers with positive antibodies to $\mathrm{Ro} / \mathrm{SSA}$ and $\mathrm{La} / \mathrm{SSB}$ groups is with accordance to results of many authors, Li et al and Izmirly et al, who reported that $63.41 \%$ and $66 \%$ respectively of NLE mothers enrolled in their studies were asymptomatic [31, 35]. However, Rivera et al reported $(15.89 \%)$ of NLE mothers with positive antibodies were asymptomatic from data obtained from Research Registry of Neonatal Lupus (RRNL), their results were lower and none contrasting with this study finding [36].

This study revealed that skin lesions in cases with positive antibodies (as seen in photos in figure 1), were distributed in head and extremities, head and trunk and whole body in $30.3 \%, 39.4 \%, 18.2 \%$ respectively, and had statistically positive correlation with positive antibodies this observation was more or less contrasting with result of Wei Sun et al who described cutaneous lesions in the form of annular-polycyclic erythematous plaques, in $51 / 54(94.44 \%)$ of their studied cutaneous NLE cases, with most affected sites, the face and head in 46 cases $(90.20 \%)$, the trunk and extremities in 39 $(76.47 \%)$ cases and $32(62.75 \%)$ cases respectively. In some cases, erythema had spread over the entire body [12]. Also, this result was with agreement with Peñate et al and Asboth et al who described skin lesions in NLE in the form of erythematous annular plaques with slight scaling, with may be urticaria-like, desquamative, occasionally ulcerative and sometimes crusted on the scalp, neck, or face with periorbital distribution, also, plaques appear on extremities or the trunk, which were more common in male than female babies [33, 34]. The cutaneous manifestations of NLE, are transient appearing after birth mostly by the age of 6 weeks and resolve spontaneously with the clearance of maternal autoantibodies from the infant's circulation by the age of 6 months, usually without any sequelae [12, 37, 38]. This study reported that cases with positive antibodies had statistically significant correlation with the use of steroid this in line with Wei Sun et al., who stated that use of topical corticosteroids was helpful in some cases of cutaneous NLE [12]. 
Percentage of positive hematologic findings in infants with positive antibodies was 13/33 (39.4\%) including anemia, lymphocytosis, neutropenia with relative lymphocytosis, eosinophilia and thrombocytopenia with percentage of $6.1 \%$, $9.1 \%, 3.0 \%, 9.1 \%$ and $12.1 \%$ respectively, and positive antibodies was statistically positively correlated with thrombocytopenia these findings are more or less similar to findings of $\mathrm{Li}$ et al, who reported in their review of NLE cases hematological abnormalities in 56 (45.5\%) cases including with thrombocytopenia, anemia and both thrombocytopenia and anemia in 17 cases $(13.82 \%)$, in 38 cases $(30.89 \%)$, and in 12 cases $(9.75 \%)$ respectively [31], although, the current study reported lymphocytosis, and not lymphopenia, this result is in agreement with Lee who stated that Lymphopenia is a relatively common in SLE adults but is not a characteristic hematologic abnormality of NLE [38]. Infants with hematological abnormalities are usually asymptomatic [39]. Autoantibodies, bind directly to the neutrophil leading to neutropenia, mainly anti Ro/SSA antibody [38]. The hematologic abnormalities (Anemia, thrombocytopenia, and neutropenia) are self-limited. However, severe thrombocytopenia if present, can lead to internal bleeding and poor prognosis.[14] Again the current study result regarding elevated Liver transaminases, alanine transaminase (ALT) and aspartate transaminase (AST) in 4 $(12.1 \%)$ cases among studied infants with statistically positive correlation with positive antibodies was more or less with agreement with results of $\mathrm{Li}$ et al, who reported $21(17.07 \%)$ cases presented with transient mild to moderate elevations of transaminases, and also, reported hyperbilirubinemia, cholestatic hepatitis and hepatomegaly in their studied NLE cases [31], but the current study did not report hyperbilirubinemia, cholestatic hepatitis or hepatomegaly. The hepatobiliary abnormalities in NLE cases occur a few weeks or months after birth and resolving thereafter [39].

In this study the $\mathrm{BW}$, length and $\mathrm{HC}$ were within normal percentiles for age but revealed statistically significant negative correlation with positive antibodies, this contrasting with result of many authors studying outcome of pregnancy in mothers with SLE who reported growth disturbances such as IUGR at birth [40 - 43], small baby for gestational age (SGA) [44 - 47], and Mokbel et al [48] who reported 22.5\% growth retardation rate where, their figure is consistent with those reported from developed countries [49 - 52].

Also, in this study, cases with positive antibodies had positive correlation with mothers rheumatologic/ autoimmune disease with positive antibodies this is contrasting with results of Friedman et al and Cimaz et al in their prospective studies of pregnancies involving mothers with anti-SSA/Ro and/ or anti-SSB/La autoantibodies reported rates of cutaneous NL of $7 \%$ and $16 \%$, respectively, both of which were higher than the $2 \%$ rates of cardiac NL $[53,9]$.

This study revealed that positive antibodies showed no statistically significant correlation with type of infant feeding either breast or formula feeding which is in concordance with study by Klauninger et al [54] who investigated levels of postnatal autoantibodies in infants born to Ro/SSA positive mothers and their correlation to NLE manifestations, by following a cohort of 32 children from birth to 1 year of age with frequent serum sampling and clinical follow-up. They reported that five of the infants developed cutaneous NLE which occurred in breastfed and in non-breastfed infants. Even if the numbers were limited, these observations are in line with this study findings.

Also, this result is contrasting with Askanase et al [55] who stated that although, breast milk from positive mothers for anti Ro/SSA and anti La/SSB antibody has been described to contain IgA and IgG specific for Ro52, Ro60 and $\mathrm{La}$, the levels of these antibodies were low or nondetectable in infants, suggesting that breast feeding was not involved in the development of skin manifestations.

The current study showed that cases with positive antibodies had no statistically significant correlation with acute phase reactants including C-reactive protein (CRP) and erythrocyte sedimentation rate (ESR) this is in line with result by Rao et al [56] in their investigation for the specificity and sensitivity of anti La/SSB antibodies to diagnose systemic lupus erythematosus (SLE) and their correlation with clinical manifestations of SLE where they reported that the levels of ESR and CRP did not differ between the positive and negative antibody cases. However their study was carried on adult and this study was on infants.

\section{Conclusion}

The studied infant cases with prolonged skin lesions with positive anti Ro/SSA and anti La/SSB antibodies with their mothers' especially asymptomatic ones should be investigated properly for NLE and development of autoimmune diseases. More than $96 \%$ of NLE patients presented with skin lesions. As NLE is a passively transferred autoimmune disease, however, NLE incidence has not been defined yet may be due to a lack of disease recognition, and the high rate of asymptomatic NLE mothers is unknown; factors like misdiagnosis of this disease, the lack of awareness as well as economic factors may play a role. Thus, pediatricians, and dermatologists, should improve their recognition of the disease to avoid misdiagnosis. Inspite of cutaneous lesions of NL are benign, it may be an important risk factor for cardiac manifestations in subsequent offspring, and a predictor for development of autoimmune disease in the infant and the mother in the long-term. So, standard protocol for diagnosis and follow up of cutaneous NLE cases and their mothers should be designed. The sample size was a limitation to this study, so, a larger study should be hold to study the predictors of cutaneous NLE.

\section{Ethics}

The present study was conducted in accordance with approval from the Ethics Committee of general organization of teaching hospital and institutes (GOTHI) and Al Galaa 
Teaching Hospital. Written informed consent was obtained from all participants mothers of infants enrolled in the study.

\section{Conflicts of Interest}

All the authors do not have any possible conflicts of interest.

\section{Abbreviations}

ALT: alanine transaminase

AST: aspartate transaminase

CHB: Congenital Heart Block

CRP: C-reactive protein

DNA: deoxy ribonucleic acid

ELISA: enzyme linked immunoassay

ESR: erythrocyte sedimentation rate

GOTHI: general organization of teaching hospital and institutes

IgG: Immunoglobulin gamma

kDa: kilo Dalton

NLE: Neonatal Lupus erythematosus

NLS: Neonatal Lupus Syndrome

RNA: ribonucleic acid

RRNL: Research Registry of Neonatal Lupus

SD: standard deviation

SLE: systemic lupus erythematosus

SPSS: statistical package for social science

SSA/Ro: Sjogren's Syndrome related antigen A (Anti-Ro Antibody)

SSB/La: Sjogren's Syndrome related antigen B (Anti-La Antibody)

UCTD: undifferentiated connective tissue disease

\section{References}

[1] Chan EK, Tan EM. Epitopic targets for autoantibodies in SLE and Sjögren's syndrome. Curr Opin Rheumatol 1989; 1:37681 .

[2] Ben-Chetrit E, Gandy BJ, Tan EM, et al. Isolation and characterization of cDNA clone encoding the $60 \mathrm{kD}$ component of the human SS-A/Ro ribonucleoprotein autoantigen. J Clin Invest 1989; 83:1284-92.

[3] O'Brien CA, Margelot K, Wolin SE. Xenopus Ro ribonucleoproteins: members of an evolutionary conserved class of cytoplasmic ribonucleoproteins. Proc Natl Acad Sci USA 1993; 90:7250-4.

[4] Meyer O. [Anti Ro/ SSA and anti La/ SSB antibodies. What's new?] 2004 Ann Med Interne (Paris). 2002 Dec; 153(8):520-9.

[5] Silverman ED, Laxer RM. Neonatal lupus erythematosus. Rheum Dis Clin North Am. 1997; 23:599-618.

[6] Dörner T, Feist E, Pruss A, Chaoui R, Göldner B, Hiepe E. Significance of autoantibodies in neonatal lupus erythematosus. Int Arch Allergy Immunol. 2000; 123:58-66.

[7] Chakravarty EF, Colón I, Langen ES, Nix DA, El-Sayed YY, et al. Factors that predict prematurity and preeclampsia in pregnancies that are complicated by systemic lupus erythematosus. Am J Obstet Gynecol. 2005; 192: 1897-1904.

[8] Nasef N, Hafez M, Bakr A. Neonatal lupus erythematosus. J Neonatol Clin Pediatr, 2014;77 (2): 82-86

[9] Cimaz R, Spence DL, Hornberger L, Silverman ED. Incidence and spectrum of neonatal lupus erythematosus: a prospective study of infants born to mothers with anti-Ro autoantibodies. J Pediatr 2003; 142: 678-83.

[10] Sontheimer RD, Maddison PJ, Reichlin M, Jordan RE, Stastny P, Gilliam JN. Serologic and HLA associations of subacute cutaneous lupus erythematosus, a clinical subset of lupus erythematosus. Ann Intern Med. 1982; 97:664-671.

[11] Weston WL, Morelli JG, Lee LA. The clinical spectrum of anti-Ro-positive cutaneous neonatal lupus erythematosus. J Am Acad Dermatol. 1999; 40:675-681.

[12] Wei Sun, Tian-Ming Yuan, Li-Hua Chen, and Hui-Min Yu: Neonatal Lupus Erythematosus: Three Case Reports and Review of the Chinese, Literature Clinical Pediatrics 2010; January 49(7) 627-634

[13] Martin V, Lee LA, Askanase AD, Katholi M, Buyon JP. Longterm follow-up of children with neonatal lupus and their unaffected siblings. Arthritis Rheum. 2002; 46: 2377-2383.

[14] Wahren-Herlenius M, Dörner T. Immunopathogenic mechanisms of systemic autoimmune disease. Lancet. 2013; 382: 819-31.

[15] Marcdante K, Kliegman RM, Behrman RE, Jenson HB. Nelson essentials of pediatrics: Elsevier Health Sciences; 2010 .

[16] Hon KL, Leung AKC. Neonatal Lupus Erythematosus. Autoimmune Dis. 2012; doi: 10. 1155/2012/301274.

[17] Lee LA. Transient autoimmunity related to maternal autoantibodies: neonatal lupus. Autoimmun Rev. 2005; 4: 207-13.

[18] Moroni G, Ponticelli C. Pregnancy in women with systemic lupus erythematosus (SLE). European journal of internal medicine. 2016; 32: 7-12.

[19] Zuppa AA, Riccardi R, Frezza S, Gallini F, Luciano RMP, Alighieri G, et al. Neonatal lupus: Follow-up in infants with anti-SSA/Ro antibodies and review of the literature. Autoimmunity Reviews. 2017. Autoimmun Rev. 2017; 16 (4):427-32.

[20] Jakobsen I, Helmig R, Stengaard-Pedersen K. Maternal and foetal outcomes in pregnant systemic lupus erythematosus patients: an incident cohort from a stable referral population followed during 1990-2010. Scandinavian journal of rheumatology. 2015;44(5):377-84.

[21] Wei Q, Ouyang Y, Zeng W, Duan L, Ge J, Liao H. Pregnancy complicating systemic lupus erythematosus: a series of 86 cases. Archives of gynecology and obstetrics. 2011; 284 (5):1067-71.

[22] Aggarwal N, Raveendran A, Suri V, Chopra S, Sikka P, Sharma A. Pregnancy outcome in systemic lupus erythematosus: Asia's largest single centre study. Archives of gynecology and obstetrics. 2011; 284 (2):281-5. 
[23] Hendawy S, Abdel-Mohsen D, Ebrahim S, Ewais H, Moussa $\mathrm{S}$, Khattab D, et al. Pregnancy related complications in patients with systemic lupus erythematosus, an Egyptian experience. Clinical medicine insights Reproductive health. 2011; 5: 17-24.

[24] Singh AG, Chowdhary VR. Pregnancy-related issues in women with systemic lupus erythematosus. International journal of rheumatic diseases. 2015; 18 (2):172-81.

[25] Chung WS, Lin CL, Chang SN, Lu CC, Kao CH. Systemic lupus erythematosus increases the risks of deep vein thrombosis and pulmonary embolism: a nationwide cohort study. Journal of Thrombosis and Haemostasis. 2014; 12 (4): 452-8.

[26] Clowse ME, Wallace DJ, Weisman M, James A, CriscioneSchreiber LG, Pisetsky DS. Predictors of preterm birth in patients with mild systemic lupus erythematosus. Annals of the rheumatic diseases. 2013; 72(9):1536-9.

[27] Zhan Z, Yang Y, Zhan Y, Chen D, Liang L, Yang X. Fetal outcomes and associated factors of adverse outcomes of pregnancy in southern Chinese women with systemic lupus erythematosus. PLoS ONE 2017; 12(4): e0176457.

[28] Karimi FZ, Saeidi M, Mirteimouri M, Maleki-Saghooni N. Maternal, Fetal and Neonatal Outcomes in Pregnant Women with Systemic Lupus Erythematosus: A Comprehensive Review Study. Int J Pediatr 2017; 5(11): 6151-71. DOI: 10. 22038/ijp. 2017. 24527. 2066

[29] Dickey BZ, Holland KE, Drolet BA, Galbraith SS, Lyon VB, Siegel DH. Demographic and clinical characteristics of cutaneous lupus erythematosus at a paediatric dermatology referral centre. Br J Dermatol. 2013 Aug. 169(2):428-33. [Medline].

[30] Dörner T, Feist E, Chaoui R, Hiepe F. Enhanced frequency of autoimmune congenital heart block in female offspring. Rheumatology (Oxford) 1999; 38, 380-2.

[31] Li YQ, Wang Q, Luo Y, Zhao Y. Neonatal lupus erythematosus: a review of 123 cases in China. Int $\mathrm{J}$ Rheum Dis. 2015; 18 (7):761-7.

[32] [32]. Buyon JP, Clancy RM. Neonatal lupus: basic research and clinical perspectives. Rheum Dis Clin North Am 2005; 31, 299-313, vii.

[33] Peñate $Y$, Luján D, Rodríguez J, Hernández-Machín B, Montenegro T, Afonso JL, et al. [Neonatal lupus erythematosus: 4 cases and clinical review]. Actas Dermosifiliogr. 2005 Dec. 96(10):690-6. [Medline].

[34] Asboth D, Kassay E, Noll J, Szalai Z. Neonatal lupus erythematosus: deep and ulcerating form. Borgyogaszati Venerol Szemle. 2000;. 76:263-5.

[35] Izmirly PM, Saxena A, Kim MY, et al. Maternal and fetal factors associated with mortality and morbidity in a multiracial/ethnic registry of anti-SSA/Ro-associated cardiac neonatal lupus. Circulation 2011; 124, 1927-35.

[36] Rivera TL, Izmirly PM, Birnbaum BK, et al. Disease progression in mothers of children enrolled in the Research Registry for Neonatal Lupus. Ann Rheum Dis 2009; 68, 82835 .

[37] Neinman AR, Lee LA, Weston WL, Buyon JB. Cutaneous manifestations of neonatal lupus without heart block: characteristics of mothers and children enrolled in a national registry. J Pediatr 2000; 142: 674-80.

[38] Lee L. A., "Cutaneous lupus in infancy and childhood," Lupus, 2010; vol. 19, no. 9, pp. 1112-1117.

[39] Silverman E. and Jaeggi E., "Non-cardiac manifestations of neonatal lupus erythematosus," Scandinavian Journal of Immunology, 2010; vol. 72, no. 3, pp. 223-225.

[40] Chen YA, Tseng J, Yang M, Tsao Y, Lin H. Acute respiratory distress syndrome in a pregnant woman with systemic lupus erythematosus: a case report. Lupus. 2014; 23: 1528-32.

[41] Buyon JP. Updates on lupus and pregnancy. Bulletin of the NYU hospital for joint diseases. 2009; 67 (3):271.

[42] Al Arfaj A, Khalil N. Pregnancy outcome in 396 pregnancies in patients with SLE in Saudi Arabia. Lupus. 2010; 19(14):1665-73.

[43] Leroux M, Desveaux C, Parcevaux M, Julliac B, Gouyon J-B, Dallay $\mathrm{D}$, et al. Impact of hydroxychloroquine on preterm delivery and intrauterine growth restriction in pregnant women with systemic lupus erythematosus: a descriptive cohort study. Lupus. 2015;24(13):1384-91

[44] Lv J, Wang W, Li Y. Clinical outcomes and predictors of fetal and maternal consequences of pregnancy in lupus nephritis patients. International urology and nephrology. 2015; 47 (8): 1379-85.

[45] de Jesus GR M-PC, de Jesus NR, dos Santos FC, Klumb EM, Carrasco MG, et al. Understanding and managing pregnancy in patients with lupus. Autoimmune diseases. 2015; 20 (15):118

[46] Nili F, McLeod L, O’Connell C, Sutton E, McMillan D. Maternal and neonatal outcomes in pregnancies complicated by systemic lupus erythematosus: a population-based study. Journal of Obstetrics and Gynaecology Canada. 2013; 35 (4):323-8.

[47] Liu. Pregnancy in women with systemic lupus erythematosus: a retrospective study of 111 pregnancies in Chinese women. 2012; 25(3):261-66.

[48] Mokbel A, Geilan AM, AboElgheit S. Could women with systemic lupus erythematosus (SLE) have successful pregnancy outcomes? Prospective observational study. Egypt Rheumatol 2013; 35(3):133-139. doi: 10. 1016/j. ejr. 2013.02 .002

[49] Surita FG, Parpinelli MA, Yonehara E, Krupa F, Cecatti JG. Systemic lupus erythematosus and pregnancy: clinical evolution, maternal and perinatal outcomes and placental findings. Sao Paulo Med J 2007; 125: 91-5.

[50] Chandran V, Aggarwal A, Misra R. Active disease during pregnancy is associated with poor fetal outcome in Indian patients with systemic lupus erythematosus. Rheumatol Int 2005; 26: 152-6.

[51] Clowse ME, Magder LS, Witter F, Petri M. The impact of increased lupus activity on obstetric outcomes. Arthritis Rheum 2005; 52: 514.

[52] Clowse ME. Lupus activity in pregnancy. Rheum Dis Clin North Am 2007; 33: 237. 
[53] Friedman DM, Kim MY, Copel JA, Davis C, Phoon CK, Glickstein JS, et al, for the PRIDE Investigators. Utility of cardiac monitoring in fetuses at risk for congenital heart block: the PR Interval and Dexamethasone Evaluation (PRIDE) prospective study. Circulation 2008; 117: 485-93.

[54] Klauninger R, Skog A, Horvath L, Winqvist O, Edner A, Bremme K, et al. Serologic follow-up of children born to mothers with Ro/SSA autoantibodies. Lupus. 2009; 18: 792 8. [PubMed]
[55] Askanase AD, Miranda-Carus ME, Tang X, Katholi M, Buyon JP. The presence of IgG antibodies reactive with components of the SSA/Ro- SSB/La complex in human breast milk: implications in neonatal lupus. Arthritis Rheum 2002; 46: 269-271.

[56] Rao L, Liu G, Li C, Li Y, Wang Z, Zhou Z, et al. Specificity of anti-SSB as a diagnostic marker for the classification of systemic lupus erythematosus. Exp Ther Med (2013) 5(6):1710-4. 10. 3892/etm. 2013. 1051 [PMC free article] [PubMed] [Cross Ref]. 